\title{
Individuelle Lernbegleitung durch Senior Teacher: erste Beobachtungen und Erfahrungen
}

Felicitas Hitz, Bruno Späti, Thomas Cerny

Es wird die männliche Form verwendet, die aber auch alle weiblichen Personen mit einschliesst.

Korrespondenz:

Dr. med. Felicitas Hitz

Kantonsspital St. Gallen Onkologie-Hämatologie CH-9007 St. Gallen

felicitas.hitz@kssg.ch

\section{Einleitung}

Nach 27-jähriger Tätigkeit als niedergelassener Onkologe und Konsiliararzt an drei Regionalspitälern gab BS seine praktische Tätigkeit Ende 2007 auf. Schon einige Zeit vor seiner Pensionierung kam die Anfrage durch Prof. Thomas Cerny, Chefarzt Onkologie-Hämatologie am Kantonsspital St. Gallen, die persönlichen Erfahrungen den onkologischen Fachassistenten als Senior Teacher weiterzugeben. Grundlage dafür war ein grosses gegenseitiges Vertrauen, das dank enger und für BS wertvoller Kooperation mit dem Kantonsspital stets gewachsen war.

Seine Motivation für diese Arbeit ergab sich aus seiner über Jahre gewonnenen Kompetenz in der Betreuung von Tumorpatienten und deren Familien. Ausserordentlich hilfreich war ihm dabei die Mitarbeit in einer Balintgruppe, die er zusammen mit praktizierenden Kollegen (Nichtonkologen) besuchte und die ihn wesentliche Elemente der Arzt-Patienten-Beziehung und deren kritische Hinterfragung in schwierigen Situationen lehrte. Diese Erfahrungen an jüngere Kollegen weiterzugeben erschien ungemein reizvoll, umso mehr als viele Kliniker und Praktiker, denen das Konzept unterbreitet wurde, davon begeistert waren und sich Ähnliches während ihrer Ausbildung ebenfalls gewünscht hätten.

BS ist nicht in den Klinikalltag eingebunden, hat keine Kaderverantwortung und ist zeitlich in seinem Engagement sehr flexibel. Sein Arbeitsvertrag limitiert seine Präsenz auf 10 Stunden in der Woche.

Das aktuelle Weiterbildungscurriculum in der Onkologie verlangt in erster Linie fachtechnisches Wissen. Kommunikative Fähigkeiten und Professionalität im Umgang mit Tumorpatienten werden wohl gefordert, aber in der Facharztprüfung nicht geprüft. Immerhin braucht es für den Facharzttitel Onkologie den Besuch eines 20-stündigen Kommunikationskurses [1]. Die Umsetzung kommunikativer Fähigkeiten im klinischen Alltag bleibt jedoch weiterhin ohne einen strukturierten Lernprozess. Diesen Fokus bietet der Senior Teacher im Rahmen supervidierter Sprechstunden im onkologischen Ambulatorium.

Literatur und allfällige Instrumente zum Assessment der Kommunikation finden sich vor allem in angelsächsischen Publikationen. Immer-

\section{Accompagnement individuel par \\ des enseignants senior: premières expériences et observations}

La communication avec les patients revêt incontestablement une importance majeure dans la formation postgraduée médicale. Sa transposition dans un concept de formation postgraduée structuré est beaucoup plus compliquée. En proposant un accompagnement individuel en la personne d'un enseignant senior, nous introduisons un modèle applicable dans la pratique clinique. L'enseignant senior est un observateur silencieux qui accompagne les consultations et en rend compte sous forme de feedback. Les premières expériences faites avec ce modèle montrent qu'il est non seulement très apprécié par tous les participants mais qu'il produit aussi un effet durable sur la façon de communiquer.

hin wurden Ansätze zur Förderung von strukturierten Lernprozessen, wie beispielsweise die Evaluation von klinischen Handlungsabläufen, auch in der Schweiz gemacht [2].

\section{Umsetzung}

Nach vorhergehender schriftlicher Aufklärung der Patienten und zusätzlicher mündlicher Einwilligung sitzt BS während der ambulanten Sprechstunde als stiller Beobachter der ArztPatienten-Interaktion im Hintergrund. Seine Beobachtung und sein Feedback richten sich auf den Umgang des Arztes mit dem Patienten, insbesondere auf die Kommunikation. Die medizinisch-onkologischen Entscheide liegen in der Verantwortung des onkologischen Kaders und werden von BS nicht in Frage gestellt oder diskutiert.

In der Regel erfolgt die Begleitung während zwei Halbtagen pro Monat. Falls vom Fachassistenten gewünscht, wird vor Beginn der Sprechstunde ein spezifischer Fokus definiert, andern- 
falls werden die Sprechstunden nach den Kriterien Gesprächsstruktur, Informationsvermittlung, Fragetyp, Kommunikation von traurigen und schlechten Nachrichten und Umgang mit Gefühlen beurteilt. Die Beobachtungen des Begleiters werden jeweils - je nach zeitlichen Verhältnissen - unmittelbar nach dem Patientenkontakt oder am Ende des Sprechstundenhalbtages mit dem angehenden Facharzt diskutiert. Der Fachassistent reflektiert seinen persönlichen Eindruck über den Patientenkontakt kritisch und nennt die gelungenen wie auch die schwierigen Elemente der Interaktion mit dem Patienten. BS kommentiert in der Folge die Selbsteinschätzung und fragt, wie eine mögliche Verbesserung in der Kommunikation hätte erreicht werden können. Die Antworten werden wiederum von BS ergänzt, wobei nicht genannte oder erkannte Schwierigkeiten angesprochen werden. Elemente, die einer vertieften Betrachtung und weiteren Trainings bedürfen, werden als Fokus für eine nächste begleitete Sprechstunde definiert.

\section{Erste Erfahrungen}

Wie kam das Projekt bei den betreuten Assistenten an? Eine erste gemeinsame Evaluation wurde nach drei Monaten vorgenommen. Es zeigte sich, dass das Konzept insgesamt auf ein gutes Echo gestossen ist. Im gemeinsamen Gespräch mit allen Beteiligten wurden die Vor- und Nachteile der supervidierten Sprechstunden diskutiert. Besonders geschätzt wurde die individuelle Betreuung über einen längeren Zeitraum. Die durch Fremdbeobachtung offenbarten Schwierigkeiten im Patientengespräch und deren Aufdeckung führten anfänglich bei den Fachassistenten zu Skepsis und Unsicherheit. Im Laufe des fortgesetzten Teachings verloren sich diese Vorbehalte aber rasch, im Gegenteil, mehrere Assistenten betonten, dass ihnen die Gegenwart von BS eine zusätzliche Sicherheit gebe. Besonders dazu beigetragen hat die gegenseitige Wertschätzung, die einen freien persönlichen Gedankenaustausch zwischen dem Fachassistenten und dem Supervisor ermöglichte. Es war erfreulich zu sehen, wie rasch Fehler im Gesprächsverhalten, wie fehlende offene Fragen, Unterbrechungen des Gesprächsflusses der Patienten, unaufmerksames Zuhören ohne offensichtliche Hinwendung und Blättern in der Krankengeschichte während des Gesprächs usw. korrigiert wurden. Diese kontinuierliche und rasche Umsetzung von eingebrachtem Feedback unterstreicht die hohe Akzeptanz des Senior Teachers.

Für den Senior Teacher bedeutet die Arbeit mit den angehenden Fachärzten eine grosse persönliche Bereicherung, einerseits wegen der praktisch vorbehaltlosen Akzeptanz seiner Arbeit, andererseits aber auch wegen der Erkenntnis, mit welchem Ernst, Fachwissen und persönlichem Bemühen um Verbesserungen sie die oft nicht leichte Arbeit mit zum Teil schwerstkranken Menschen leisten. Die Rolle des lernenden Arztes ist schwierig, da er sowohl die Erwartungen des Kaders erfüllen muss als auch für viele Patienten schon als «vollwertiger Arzt» gilt, der zum Teil grosses Vertrauen geniesst und grossen Ansprüchen gerecht werden muss. Die oft verborgenen Sorgen und Ängste zu diskutieren und in den Kontext einer langen persönlichen Erfahrung zu stellen - ohne die individuelle Arbeitsweise des Assistenten zu beinträchtigen - sowie die Begleitung über einen längeren Zeitraum, mit der Möglichkeit, Entwicklungen zu beobachten, ist beglückend und motivierend.

Wichtig war uns auch die Akzeptanz der Patienten. Eine anonyme Umfrage bestätigte dies bei allen Betroffenen. In keinem Fall haben wir es erlebt, dass sie die Präsenz von BS während der Sprechstunde ablehnten. Im Gegenteil, das Projekt wurde von vielen Patienten sehr wohlwollend angenommen und unterstützt.

Die ersten Auswertungen der supervidierten Sprechstunden basieren auf subjektiven Eindrücken der Fachassistenten und des Senior Teachers sowie der Patienten. In einer nächsten Projektphase möchten wir den longitudinalen Effekt der individuellen Lernbegleitung auf das Kommunikationsverhalten erfassen. Die bisherige qualitative Beurteilung soll mit validierten Assessmentinstrumenten der Kommunikation erfasst werden und eine quantifizierbare Beurteilung ermöglichen.

\section{Diskussion}

Senior Teaching im Rahmen von strukturierten Lernprozessen bietet einen mehrfachen Nutzen für alle Beteiligten. Die wertvollen Kompetenzen langjähriger Berufserfahrung können an junge Kollegen weitergegeben werden und sind kein verlorenes Gut beim Eintritt ins Pensionsalter. Im Gegensatz zu einem Kaderarzt ist der Senior Teacher nur für die ihm zugeteilte Aufgabe angestellt und muss sich nicht zwischen Telefonaten, Sitzungen und klinischer Arbeit auch noch um gutes Teaching kümmern. Der Bildungsauftrag der Kaderärzte wird mit diesem Modell in einem Spezialbereich ergänzt, aber nicht ersetzt. Für eine wirksame Verbesserung der Patientenkommunikation drängt sich das Senior-Teaching-Modell geradezu auf, da unseres Erachtens nur viele Patientengespräche, über einen längeren Zeitraum beobachtet, und nach jeweiliger gemeinsamer Reflexion zu einem veränderten Verhalten führen. 
Diesen Zeitaufwand kann in der Regel kein klinisch tätiger Kaderarzt leisten. Deswegen findet die Verbesserung der Kommunikation im Klinikalltag selten genügend Aufmerksamkeit, obwohl ihre grosse Bedeutung kaum bestritten wird.

Individuelle Lernbegleitung wird nicht nur hochgeschätzt, wie unsere ersten Erfahrungen belegen, sondern ist auch eine sehr effektive Form des Lernens [3]. Die unmittelbare Rückmeldung durch den Beobachter und das gemeinsame Reflektieren der praktischen Tätigkeit haben eine nachhaltige Wirkung auf das ärztliche Verhalten. Dies im Gegensatz zu Vorlesungen, die in einer kurzen Zeitspanne eine hohe Dichte an Wissen vermitteln, jedoch keinen guten Lerneffekt erzielen. Die Vorlesung dient dem Vermitteln des Grundwissens, während Gruppenarbeiten und strukturiertes Feedback im Rahmen von supervidierten Sprechstunden - wie in unserem Fall theoretisches Wissen mit Handeln verknüpfen.

Durch die Einführung der individuellen Lernbegleitung konnte eine zusätzliche Modalität geschaffen werden, die das bereits existierende Weiterbildungsangebot ergänzt. Die Kombination verschiedener Lernstrategien führt zu einer optimalen Verankerung der Informationen im Gedächtnis und damit zu einem hohen Umsetzungsgrad des theoretischen Wissens im Alltag. Obwohl die Methode eine aufwendige Form des Lernens ist, wenn man berücksichtigt, dass pro Zeiteinheit jeweils nur eine Person profitieren kann, ist doch ihre Wirksamkeit in Bezug auf eine Verhaltensänderung deutlich höher einzustufen als Vorlesungen oder Seminare.

Die kontinuierliche Supervision über einen längeren Zeitraum schafft auch eine Basis des Vertrauens zwischen Lernenden und Beobachter. Diese ermöglicht in einer unbelasteten Beziehung auch die kritische Reflexion der Arzt-PatientenInteraktion, eine wichtige, im hektischen Klinikalltag kaum mögliche Diskussion über den Umgang und die Kommunikation mit dem Patienten. Die Fähigkeit, sich selbst einzuschätzen und sein eigenes Verhalten zu überdenken, bedeutet einen hohen Grad an Professionalität, eine Kompetenz, die für jeden Arzt während der Zeit seiner praktischen Tätigkeit unverzichtbar ist.

Der Senior Teacher als individueller Lernbegleiter hat sich an unserer Institution einen hohen Beliebtheitsgrad geschaffen. Wir werden uns diesen Luxus auch weiterhin leisten!

\section{Literatur}

1 Weiterbildungsprogramm Facharzt für Medizinische Onkologie.

2 Berendonk C, Beyeler C, Westkämper R, Giger M. Strukturiertes Feedback in der ärztlichen Weiterbildung: Mini-CEX und DOPS. Schweiz Ärztezeitung. 2008;89(32):1337-40.

3 Gordon J. ABC of learning and teaching in medicine: one to one teaching and feedback. BMJ. 2003;326:543-5. 